\title{
Potential Theory of Special Subordinators and Subordinate Killed Stable Processes
}

\author{
Renming Song,,$^{1,3, *}$ and Zoran Vondraček ${ }^{2, \dagger}$
}

Received March 17, 2005; revised May 25, 2005

\begin{abstract}
In this paper we introduce a large class of subordinators called special subordinators and study their potential theory. Then we study the potential theory of processes obtained by subordinating a killed symmetric stable process in a bounded open set $D$ with special subordinators. We establish a one-to-one correspondence between the nonnegative harmonic functions of the killed symmetric stable process and the nonnegative harmonic functions of the subordinate killed symmetric stable process. We show that nonnegative harmonic functions of the subordinate killed symmetric stable process are continuous and satisfy a Harnack inequality. We then show that, when $D$ is a bounded $\kappa$-fat set, both the Martin boundary and the minimal Martin boundary of the subordinate killed symmetric stable process in $D$ coincide with the Euclidean boundary $\partial D$.
\end{abstract}

KEY WORDS: Killed Brownian motions; killed symmetric stable processes; subordinators; Bernstein functions; complete Bernstein functions; subordination; harmonic functions; Green function; Martin kernel; Martin boundary; Harnack inequality.

AMS 2000 Mathematics Subject Classifications: Primary 60J45; Secondary $60 \mathrm{~J} 75 ; 31 \mathrm{C} 05 ; 31 \mathrm{C} 35$.

\footnotetext{
${ }^{1}$ Department of Mathematics, University of Illinois, Urbana, IL 61801, USA

E-mail: rsong@math.uiuc.edu

${ }^{2}$ Department of Mathematics, University of Zagreb, Zagreb, Croatia

E-mail: vondra@math.hr

${ }^{3}$ To whom the correspondence should be addressed.

* The research of this author is supported in part by a joint US-Croatia grant INT 0302167.

†The research of this author is supported in part by MZOS grant 0037107 of the Republic of Croatia and in part by a joint US-Croatia grant INT 0302167.
} 


\section{INTRODUCTION}

Let $D$ be a bounded open set in $\mathbb{R}^{d}, d \geq 3$, and let $\left.\Delta\right|_{D}$ be the Dirichlet Laplacian in $D$. This operator is the infinitesimal generator of the semigroup $\left(P_{t}^{D}: t \geq 0\right)$ corresponding to the process $X^{D}=\left(X_{t}^{D}: t \geq\right.$ 0 ), the Brownian motion killed upon exiting $D$. Let $S=\left(S_{t}: t \geq 0\right)$ be an $\alpha / 2$-stable subordinator independent of $X^{D}$, where $0<\alpha<2$, and let $Z_{\alpha}^{D}=\left(Z_{\alpha}^{D}(t): t \geq 0\right)$ be the process $X^{D}$ subordinate by $S: Z_{\alpha}^{D}(t):=X^{D}\left(S_{t}\right)$. The infinitesimal generator of the semigroup of $Z_{\alpha}^{D}$ is the fractional power $-\left(-\left.\Delta\right|_{D}\right)^{\alpha / 2}$ of the negative Dirichlet Laplacian. Despite the importance of this operator in analysis, the probabilistic and potential-theoretic properties of the corresponding process $Z_{\alpha}^{D}$ began to be studied only recently. The study of the process $Z_{\alpha}^{D}$ was initiated in Ref. 16. In Ref. 18 (see also Ref. 14) the domain of the Dirichlet form of $Z_{\alpha}^{D}$ was identified when $D$ is a bounded smooth domain and $\alpha \neq 1$. In Ref. 24 and Ref. 23, the process $Z_{\alpha}^{D}$ was studied in detail and sharp upper and lower bounds on the jumping function and the Green function of $Z_{\alpha}^{D}$ were established when $D$ is a bounded $C^{1,1}$ domain.

One of the most intriguing aspects of the potential theory of $Z_{\alpha}^{D}$ was discovered in Ref. 16, and completely described in Ref. 15. Let us introduce another subordinate process, $Z_{2-\alpha}^{D}$, obtained by subordinating killed Brownian motion $X^{D}$ by an independent $(1-\alpha / 2)$-stable subordinator. Let $G^{D}, G_{\alpha}^{D}$ and $G_{2-\alpha}^{D}$ denote the potential operators of $X^{D}, Z_{\alpha}^{D}$ and $Z_{2-\alpha}^{D}$, respectively. Then the following factorization identity holds true:

$$
G^{D}=G_{\alpha}^{D} G_{2-\alpha}^{D}=G_{2-\alpha}^{D} G_{\alpha}^{D} .
$$

If it is assumed that the semigroup $\left(P_{t}^{D}: t \geq 0\right)$ of $X^{D}$ is intrinsically ultracontractive (a rather mild assumption on the domain $D$ ), then (1.1) has the following important consequence: the operator $G_{\alpha}^{D}$ is a oneto-one mapping from the set of excessive (respectively, nonnegative harmonic) functions of $Z_{2-\alpha}^{D}$ onto the set of excessive (respectively, nonnegative harmonic) functions of $X^{D}$. Moreover, the inverse mapping is given by the following explicit formula:

$$
\left(G_{\alpha}^{D}\right)^{-1} s(x)=\frac{\alpha}{2 \Gamma(1-\alpha / 2)} \int_{0}^{\infty} t^{-\alpha / 2-1}\left(s(x)-P_{t}^{D} s(x)\right) d t,
$$

where $s$ is excessive (respectively, nonnegative harmonic) for $X^{D}$. This formula is used to prove that all nonnegative harmonic functions of $Z_{\alpha}^{D}$ are continuous, which together with (1.1) and the intrinsic ultracontractivity of $\left(P_{t}^{D}\right)$ enables a novel proof of Harnack inequality. Another consequence of (1.2) is the identification of the Martin boundary with respect to $Z_{\alpha}^{D}$ of a Lipschitz domain $D$ with its Euclidean boundary $\partial D$. 
The Laplace exponent of the $\alpha / 2$-stable subordinator is $\phi(\lambda)=$ $\lambda^{\alpha / 2}, \lambda>0$. Clearly, $\lambda / \lambda^{\alpha / 2}=\lambda^{1-\alpha / 2}$ is the Laplace exponent of the $(1-$ $\alpha / 2$ )-stable subordinator. This existence of a "dual" subordinator is the key for the factorization (1.1). Motivated by this fact, we introduce in this paper subordinators whose Laplace exponent $\phi(\lambda)$ has the property that $\lambda / \phi(\lambda)$ is again the Laplace exponent of a subordinator. We call such subordinators special, and argue in Section 2 that they comprise a large subclass of subordinators. We show that special subordinators can be characterized by the following very useful property: a subordinator $S=$ $\left(S_{t}, t \geq 0\right)$ is special if and only if its potential measure restricted to $(0, \infty)$ has a decreasing density.

The main contribution of this paper, besides introducing the concepts of special Bernstein functions and special subordinators, is the realization that the key point for the main results of Ref. 16 and Ref. 15, is the fact that stable subordinators are special. We will show in this paper that the main results of Ref. 16 and Ref. 15, remain valid for the killed Brownian motion $X^{D}$ subordinate by a special subordinator with infinite Lévy measure or positive drift (or both). The resulting class of subordinate processes is a significant extension of the one studied in Ref. 15. In particular, this class contains discontinuous processes with a continuous component. Moreover, if the Lévy measure of the subordinator is finite, the jumping times of the subordinate process will be discrete. The Harnack inequality that we prove for nonnegative harmonic functions of such processes is to the best of our knowledge the first one in the literature.

Another generalization that we introduce consists of replacing the underlying killed Brownian motion $X^{D}$ by a rotationally invariant $\alpha$-stable process, $0<\alpha \leq 2$, killed upon exiting $D$. For $0<\alpha<2$, this process is discontinuous which introduces some technical, but not essential, difficulties. For simplicity, from now on we will use the term symmetric $\alpha$-stable process, instead of the more precise one - rotationally invariant. So, the process that we are going to study is the symmetric $\alpha$-stable process killed upon exiting $D$, subordinate by a special subordinator with infinite Lévy measure or positive drift.

The content of this paper is organized as follows. In Section 2 we first introduce the concepts of special Bernstein functions and special subordinators, show that this class is large and contains most of the known subordinators. Then we study some potential theoretical properties of special subordinators. In particular, we characterize special subordinators in terms of their potential measures. In Section 3 we introduce killed symmetric stable processes in a bounded open set subordinate by special subordinators "dual" to each other. One of the subordinators is assumed to have an infinite Lévy measure or positive drift, while the other subordinator may be 
a compound Poisson process. Clearly, these two subordinate processes do not have symmetric roles. We are interested in the potential theory of the process $X^{D}$ subordinate by special subordinators having an infinite Lévy measure or positive drift. The main result that we establish is a one-to-one correspondence between the family of excessive (respectively nonnegative harmonic) functions of killed symmetric stable processes and the family of excessive (respectively nonnegative harmonic) functions of the subordinate process. We are in particular interested in nonnegative harmonic functions of the subordinate process. We prove that they are continuous and present the Harnack inequality. In Section 4 we show that when $D$ is a bounded $\kappa$-fat set, the Martin boundary and minimal Martin boundary of the subordinate killed symmetric stable process both coincide with the Euclidean boundary $\partial D$.

In the remainder of this section we shall recall the definitions of harmonic functions and excessive functions with respect to a standard process $X=\left(X_{t}, \mathbb{P}_{x}\right)$ in a domain $D$ in $\mathbb{R}^{d}$. A Borel function $h$ on $D$ is said to be harmonic with respect to $X$ if $h$ is not identically infinite in $D$ and if for every relatively compact open subset $U \subset \bar{U} \subset D$,

$$
h(x)=\mathbb{E}_{x}\left[h\left(X\left(\tau_{U}\right)\right)\right], \quad \forall x \in U,
$$

where $\tau_{U}=\inf \left\{t: X_{t} \notin U\right\}$ is the first exit time of $U$. We are going to use $\mathcal{H}(\mathrm{X})$ to denote the collection of all the functions on $D$ which are harmonic with respect to $X$ and $\mathcal{H}^{+}(X)$ to denote the collection of all the nonnegative functions on $D$ which are harmonic with respect to $X$. A nonnegative function which is not identically infinite on $D$ is said to be excessive with respect to $X$ if (i) $\mathbb{E}_{x}\left[f\left(X_{t}\right)\right] \leq f(x)$ for every $t>0$ and every $x \in D$; and (ii) $\lim _{t \downarrow 0} \mathbb{E}_{x}\left[f\left(X_{t}\right)\right]=f(x)$ for every $x \in D$. We are going to use $\mathcal{S}(X)$ to denote the collection of all the excessive functions with respect to $X$. It is well known that $\mathcal{H}^{+}(X) \subset \mathcal{S}(X)$.

\section{SPECIAL SUBORDINATORS AND COMPLETE BERNSTEIN FUNCTIONS}

Let $S=\left(S_{t}: t \geq 0\right)$ be a subordinator, that is, an increasing Lévy process taking values in $[0, \infty]$ with $S_{0}=0$. We remark that our subordinators are what some authors call killed subordinators. The Laplace transform of the law of $S$ is given by the formula

$$
\mathbb{E}\left[\exp \left(-\lambda S_{t}\right)\right]=\exp (-t \phi(\lambda)), \quad \lambda>0 .
$$


The function $\phi:(0, \infty) \rightarrow \mathbb{R}$ is called the Laplace exponent of $S$, and it can be written in the form

$$
\phi(\lambda)=a+b \lambda+\int_{0}^{\infty}\left(1-e^{-\lambda t}\right) \mu(d t) .
$$

Here $a, b \geq 0$, and $\mu$ is a $\sigma$-finite measure on $(0, \infty)$ satisfying

$$
\int_{0}^{\infty}(t \wedge 1) \mu(d t)<\infty .
$$

The constant $a$ is called the killing rate, $b$ the drift, and $\mu$ the Lévy measure of the subordinator $S$. By using condition (2.3) above one can easily check that

$$
\begin{aligned}
& \lim _{t \rightarrow 0} t \mu(t, \infty)=0 \\
& \int_{0}^{1} \mu(t, \infty) d t<\infty .
\end{aligned}
$$

Recall that a $C^{\infty}$ function $\phi:(0, \infty) \rightarrow[0, \infty)$ is called a Bernstein function if $(-1)^{n} D^{n} \phi \leq 0$ for every $n \in \mathbb{N}$. It is well known that a function $\phi:(0, \infty) \rightarrow \mathbb{R}$ is a Bernstein function if and only if it has the representation given by (2.2).

We now introduce the concepts of special Bernstein functions and special subordinators.

Definition 2.1. A Bernstein function $\phi$ is called a special Bernstein function if $\lambda / \phi(\lambda)$ is also a Bernstein function. A subordinator $S$ is called a special subordinator if its Laplace exponent is a special Bernstein function.

Special subordinators occur naturally in various situations. For instance, they appear as the ladder time process for a Lévy process which is not a compound Poisson process, see page 166 of Ref. 3. Yet another situation in which they appear naturally is in connection with the exponential functional of subordinators (see Ref. 4).

Our prime example of special Bernstein functions are complete Bernstein functions, also called operator monotone functions in some literature. A function $\phi:(0, \infty) \rightarrow \mathbb{R}$ is called a complete Bernstein function if there exists a Bernstein function $\eta$ such that

$$
\phi(\lambda)=\lambda^{2} \mathcal{L} \eta(\lambda), \quad \lambda>0,
$$


where $\mathcal{L}$ stands for the Laplace transform. It is known (see, for instance, Remark 3.9.28 and Theorem 3.9.29 of Ref. 17) that every complete Bernstein function is a Bernstein function and that the following three conditions are equivalent:

(i) $\phi$ is a complete Bernstein function;

(ii) $\psi(\lambda):=\lambda / \phi(\lambda)$ is a complete Bernstein function;

(iii) $\phi$ is a Bernstein function whose Lévy measure $\mu$ is given by

$$
\mu(d t)=\int_{0}^{\infty} \mathrm{e}^{-s t} \gamma(d s) d t
$$

where $\gamma$ is a measure on $(0, \infty)$ satisfying

$$
\int_{0}^{1} \frac{1}{s} \gamma(d s)+\int_{1}^{\infty} \frac{1}{s^{2}} \gamma(d s)<\infty .
$$

The equivalence of (i) and (ii) says that every complete Bernstein function is a special Bernstein function. Note also that it follows from the condition (iii) above that being a complete Bernstein function only depends on the Lévy measure and that the Lévy measure $\mu(d t)$ of any complete Bernstein function has a completely monotone density.

The family of all complete Bernstein functions is a closed convex cone containing positive constants. The following properties of complete Bernstein functions are well known, see, for instance, Ref. 20: (i) If $\phi$ is a nonzero complete Bernstein function, then so are $\phi\left(\lambda^{-1}\right)^{-1}$ and $\lambda \phi\left(\lambda^{-1}\right)$; (ii) if $\phi_{1}$ and $\phi_{2}$ are nonzero complete Bernstein functions and $\beta \in(0,1)$, then $\phi_{1}^{\beta}(\lambda) \phi_{2}^{1-\beta}(\lambda)$ is also a complete Bernstein function; (iii) if $\phi_{1}$ and $\phi_{2}$ are nonzero complete Bernstein functions and $\beta \in(-1,0) \cup(0,1)$, then $\left(\phi_{1}^{\beta}(\lambda)+\phi_{2}^{\beta}(\lambda)\right)^{1 / \beta}$ is also a complete Bernstein function.

Recall that a probability distribution function on $[0, \infty)$ is called a generalized Gamma convolution (GGC for short) if it is infinitely divisible and its Lévy measure has a density $l$ such that the function

$$
x \rightarrow x l(x), \quad x>0
$$

is completely monotone. The class of GGC distribution can be characterized as the smallest class of distributions on $[0, \infty)$ that contains the Gamma distributions and is closed with respect to convolutions and weak limits. It is known that if a probability distribution function on $[0, \infty)$ is a GGC, then its Laplace exponent must be a complete Bernstein function. For these and other results about GGC, please see Ref. 8.

Most of the familiar Bernstein functions are complete Bernstein functions. The following are some examples of complete Bernstein functions 
(Ref. 17): (i) $\lambda^{\alpha}, \alpha \in(0,1]$; (ii) $(\lambda+1)^{\alpha}-1, \alpha \in(0,1)$; (iii) $\log (1+\lambda)$; (iv) $\frac{\lambda}{\lambda+1}$. The first family corresponds to $\alpha$-stable subordinators $(0<\alpha<1)$, and pure drift $(\alpha=1)$, the second family corresponds to relativistic $\alpha$-stable subordinators, and the third corresponds to the gamma subordinator. The distributions corresponding to the complete Bernstein functions in the first three families are GGC. An example of a Bernstein function which is not a complete Bernstein function is $1-\mathrm{e}^{-\lambda}$. One can also check that $1-\mathrm{e}^{-\lambda}$ is not a special Bernstein function as well.

After showing that the family of special Bernstein functions is indeed large and that it contains other important classes of Bernstein functions from the literature, we come back to the main development of this section. The potential measure of the subordinator $S$ is defined by

$$
U(A)=\mathbb{E} \int_{0}^{\infty} 1_{\left(S_{t} \in A\right)} d t
$$

and its Laplace transform is given by

$$
\mathcal{L} U(\lambda)=\int_{0}^{\infty} e^{-\lambda t} d U(t)=\mathbb{E} \int_{0}^{\infty} \exp \left(-\lambda S_{t}\right) d t=\frac{1}{\phi(\lambda)} .
$$

We are going to derive a characterization of special subordinators in terms of their potential measures. Roughly, a subordinator $S$ is special if and only if its potential measure $U$ restricted to $(0, \infty)$ has a decreasing density. To be more precise, let $S$ be a special subordinator with the Laplace exponent $\phi$ given by

$$
\phi(\lambda)=a+b \lambda+\int_{0}^{\infty}\left(1-e^{-\lambda t}\right) \mu(d t) .
$$

Then

$$
\begin{aligned}
\lim _{\lambda \rightarrow 0} \frac{\lambda}{\phi(\lambda)} & =\left\{\begin{array}{cc}
0, & a>0 \\
\frac{1}{b+\int_{0}^{\infty} t \mu(d t)}, & a=0
\end{array}\right. \\
\lim _{\lambda \rightarrow \infty} \frac{1}{\phi(\lambda)} & =\left\{\begin{array}{cc}
0, & b>0 \text { or } \mu(0, \infty)=\infty \\
\frac{1}{a+\mu(0, \infty)}, & b=0 \text { and } \mu(0, \infty)<\infty .
\end{array}\right.
\end{aligned}
$$

Since $\lambda / \phi(\lambda)$ is a Bernstein function, we must have

$$
\frac{\lambda}{\phi(\lambda)}=\tilde{a}+\tilde{b} \lambda+\int_{0}^{\infty}\left(1-e^{-\lambda t}\right) v(d t)
$$


for some Lévy measure $v$, and

$$
\begin{aligned}
& \tilde{a}=\left\{\begin{array}{cc}
0, & a>0 \\
\frac{1}{b+\int_{0}^{\infty} t \mu(d t)}, & a=0
\end{array}\right. \\
& \tilde{b}=\left\{\begin{array}{cl}
0, & b>0 \text { or } \mu(0, \infty)=\infty \\
\frac{1}{a+\mu(0, \infty)}, & b=0 \text { and } \mu(0, \infty)<\infty .
\end{array}\right.
\end{aligned}
$$

Equivalently,

$$
\frac{1}{\phi(\lambda)}=\tilde{b}+\int_{0}^{\infty} e^{-\lambda t} \tilde{\Pi}(t) d t
$$

with

$$
\tilde{\Pi}(t)=\tilde{a}+v(t, \infty), \quad t>0 .
$$

Let $\tau(d t):=\tilde{b} \epsilon_{0}(d t)+\tilde{\Pi}(t) d t$. Then the right-hand side in (2.11) is the Laplace transform of the measure $\tau$. Since $1 / \phi(\lambda)=\mathcal{L} U(\lambda)$, the Laplace transform of the potential measure $U$ of $S$, we have that

$$
\mathcal{L} U(\lambda)=\mathcal{L} \tau(\lambda) .
$$

Therefore,

$$
U(d t)=\tilde{b} \epsilon_{0}(d t)+u(t) d t,
$$

with a decreasing function $u(t)=\tilde{\Pi}(t)$.

Conversely, suppose that $S$ is a subordinator with potential measure given by

$$
U(d t)=c \epsilon_{0}(d t)+u(t) d t
$$

for some $c \geq 0$ and some decreasing function $u:(0, \infty) \rightarrow(0, \infty)$ satisfying $\int_{0}^{1} u(t) d t<\infty$. Then

$$
\frac{1}{\phi(\lambda)}=\mathcal{L} U(\lambda)=c+\int_{0}^{\infty} e^{-\lambda t} u(t) d t
$$

It follows that

$$
\begin{aligned}
\frac{\lambda}{\phi(\lambda)} & =c \lambda+\int_{0}^{\infty} u(t) d\left(1-e^{-\lambda t}\right) \\
& =c \lambda+\left.u(t)\left(1-e^{-\lambda t}\right)\right|_{0} ^{\infty}-\int_{0}^{\infty}\left(1-e^{-\lambda t}\right) u(d t) \\
& =c \lambda+u(\infty)+\int_{0}^{\infty}\left(1-e^{-\lambda t}\right) \gamma(d t),
\end{aligned}
$$


with $\gamma(d t)=-u(d t)$. In the last equality we used that $\lim _{t \rightarrow 0} u(t)\left(1-e^{-\lambda t}\right)=0$. This is a consequence of the assumption $\int_{0}^{1} u(t) d t<\infty$. It is easy to check, by using the same integrability condition on $u$, that $\int_{0}^{\infty}(1 \wedge t) \gamma(d t)<\infty$, so that $\gamma$ is a Lévy measure. Therefore, $\lambda / \phi(\lambda)$ is a Bernstein function, implying that $S$ is a special subordinator.

In this way we have proved the following

Theorem 2.1. Let $S$ be a subordinator with potential measure $U$. Then $S$ is special if and only if

$$
U(d t)=c \epsilon_{0}(d t)+u(t) d t
$$

for some $c \geq 0$ and some decreasing function $u:(0, \infty) \rightarrow(0, \infty)$ satisfying $\int_{0}^{1} u(t) d t<\infty$.

Remark 2.2. Note that from the proof above we have the explicit form of the density $u: u(t)=\tilde{\Pi}(t)$ where $\tilde{\Pi}(t)=\tilde{a}+v(t, \infty)$. Here $v$ is the Lévy measure of $\lambda / \phi(\lambda)$. In case when $\phi(\lambda)$ (and therefore also $\lambda / \phi(\lambda)$ ) is a complete Bernstein function, it follows from the property (iii) of complete Bernstein function that the tail $t \mapsto v(t, \infty)$ of Lévy measure $v$ is a complete monotone function. Therefore, the potential density $u$ of $S$ is also completely monotone. This was first proved in Ref. 21.

Note that by comparing expressions (2.8) and (2.12) for $\lambda / \phi(\lambda)$, and by using formulae (2.9) and (2.10), it immediately follows that

$$
\begin{aligned}
c & =\tilde{b}=\left\{\begin{array}{cc}
0, & b>0 \text { or } \mu(0, \infty)=\infty \\
\frac{1}{a+\mu(0, \infty)}, & b=0 \text { and } \mu(0, \infty)<\infty
\end{array}\right. \\
u(\infty) & =\tilde{a}=\left\{\begin{array}{cl}
0, & a>0 \\
\frac{1}{b+\int_{0}^{\infty} t \mu(d t)}, & a=0
\end{array}\right. \\
u(t) & =\tilde{a}+v(t, \infty) .
\end{aligned}
$$

In particular, it cannot happen that both $a$ and $\tilde{a}$ are positive, and similarly, that both $b$ and $\tilde{b}$ are positive. Moreover, it is clear from the definition of $\tilde{b}$ that $\tilde{b}>0$ if and only if $b=0$ and $\mu(0, \infty)<\infty$.

We record now some consequences of Theorem 2.1 and the formulae above.

Corollary 2.3. Suppose that $S=\left(S_{t}: t \geq 0\right)$ is a subordinator whose Laplace exponent

$$
\phi(\lambda)=a+b \lambda+\int_{0}^{\infty}\left(1-e^{-\lambda t}\right) \mu(d t)
$$


is a special Bernstein function with $b>0$ or $\mu(0, \infty)=\infty$. Then the potential measure $U$ of $S$ has a decreasing density $u$ satisfying

$$
\begin{aligned}
\lim _{t \rightarrow 0} t u(t) & =0 \\
\lim _{t \rightarrow 0} \int_{0}^{t} s d u(s) & =0 .
\end{aligned}
$$

Proof. The formulae follow immediately from $u(t)=\tilde{a}+v(t, \infty)$ and (2.4), (2.5) applied to $v$.

Corollary 2.4. Suppose that $S=\left(S_{t}: t \geq 0\right)$ is a special subordinator with the Laplace exponent given by

$$
\phi(\lambda)=a+\int_{0}^{\infty}\left(1-e^{-\lambda t}\right) \mu(d t)
$$

where $\mu$ satisfies $\mu(0, \infty)=\infty$. Then

$$
\psi(\lambda):=\frac{\lambda}{\phi(\lambda)}=\tilde{a}+\int_{0}^{\infty}\left(1-e^{-\lambda t}\right) v(d t)
$$

where the Lévy measure $v$ satisfies $v(0, \infty)=\infty$.

Let $T$ be the subordinator with the Laplace exponent $\psi$. If $u$ and $v$ denote the potential density of $S$ and $T$ respectively, then

$$
v(t)=a+\mu(t, \infty) .
$$

In particular, $a=v(\infty)$ and $\tilde{a}=u(\infty)$. Moreover, $a$ and $\tilde{a}$ cannot be both positive.

In the rest of the paper we will assume that $\phi$ is a special Bernstein function with the representation (2.2) where $b>0$ or $\mu(0, \infty)=\infty$. Let $S$ be a subordinator with the Laplace exponent $\phi$, and let $U$ denote its potential measure. By Corollary 2.3, $U$ has a decreasing density $u$ : $(0, \infty) \rightarrow(0, \infty)$. Let $T$ be a subordinator with the Laplace exponent $\psi(\lambda)=\lambda / \phi(\lambda)$ and let $V$ denote its potential measure. Then $V(d t)=$ $b \epsilon_{0}(d t)+v(t) d t$ where $v:(0, \infty) \rightarrow(0, \infty)$ is a decreasing function. If $b>0$, the potential measure $V$ has an atom at zero, and hence the subordinator $T$ is a compound Poisson process (this can be also seen as follows: since $b>0$, we have $u(0+)<\infty$, and hence $v(0, \infty)=u(0+)-\tilde{a}<\infty)$. Note that in case $b>0$, the Lévy measure $\mu$ can be finite. If $b=0$, we require that $\mu(0, \infty)=\infty$, and then, by Corollary $2.4, \psi(\lambda)=\lambda / \phi(\lambda)$ has the same 
form as $\phi$, namely $\tilde{b}=0$ and $v(0, \infty)=\infty$. In this case, subordinators $S$ and $T$ play symmetric roles.

The following result is crucial for the development in the remainder of this paper.

Theorem 2.5. Let $\phi$ be a special Bernstein function with representation (2.2) satisfying $b>0$ or $\mu(0, \infty)=\infty$. Then

$$
b u(t)+\int_{0}^{t} u(s) v(t-s) d s=b u(t)+\int_{0}^{t} v(s) u(t-s) d s=1, \quad t>0 .
$$

Proof. Since for all $\lambda>0$ we have

$$
\begin{aligned}
& \frac{1}{\phi(\lambda)}=\mathcal{L} u(\lambda) \\
& \frac{\phi(\lambda)}{\lambda}=b+\mathcal{L} v(\lambda)
\end{aligned}
$$

after multiplying we get

$$
\begin{aligned}
\frac{1}{\lambda} & =b \mathcal{L} u(\lambda)+\mathcal{L} u(\lambda) \mathcal{L} v(\lambda) \\
& =b \mathcal{L} u(\lambda)+\mathcal{L}(u * v)(\lambda) .
\end{aligned}
$$

Inverting this equality gives

$$
1=b u(t)+\int_{0}^{t} u(s) v(t-s) d s, \quad t>0 .
$$

\section{NONNEGATIVE HARMONIC FUNCTIONS OF THE SUBORDINATE PROCESS}

Suppose that $\alpha \in(0,2]$ and let $X=\left(X_{t}: t \geq 0\right)$ be a symmetric $\alpha$-stable process in $\mathbb{R}^{d}$ with characteristic function

$$
\mathbb{E}\left[\exp \left(i \xi \cdot\left(X_{t}-X_{0}\right)\right)\right]=e^{-t|\xi|^{\alpha}}, \quad \xi \in \mathbb{R}^{d}, t \geq 0 .
$$

Let $D$ be a bounded open set in $\mathbb{R}^{d}$ which is further assumed to be connected when $\alpha=2$, and let $\tau_{D}=\inf \left\{t>0: X_{t} \notin D\right\}$ be the exit time of $X$ from $D$. Define

$$
X_{t}^{D}= \begin{cases}X_{t}, & t<\tau_{D} \\ \partial, & t \geq \tau_{D}\end{cases}
$$


where $\partial$ is the cemetery. We call $X^{D}$ the symmetric $\alpha$-stable process killed upon exiting $D$. The semigroup of $X^{D}$ will be denoted by $\left(P_{t}^{D}\right)_{t \geq 0}$, and its transition density by $p^{D}(t, x, y), t \geq 0, x, y \in \mathbb{R}^{d}$. The transition density $p^{D}(t, x, y)$ is strictly positive, and hence the eigenfunction $\varphi_{0}$ of the operator $\left.(-\Delta)^{\frac{\alpha}{2}}\right|_{D}$ corresponding to the smallest eigenvalue $\lambda_{0}$ can be chosen to be strictly positive, see, for instance, Ref. 11 . The potential operator of $X^{D}$ is given by

$$
G^{D} f(x)=\int_{0}^{\infty} P_{t}^{D} f(x) d t,
$$

and has a density $G^{D}(x, y), x, y \in D$. Here, and further below, $f$ denotes a nonnegative Borel function on $D$. The potential theory of the killed symmetric $\alpha$-stable process, $0<\alpha<2$, has been studied extensively in the late nineties. We will need the following two facts: If $h$ is a nonnegative harmonic function for $X^{D}$, then $h$ and $P_{t}^{D} h$ are continuous in $D$. The continuity of harmonic functions is proved in Ref. 7. To show the second fact, note that from the explicit formula for the Poisson kernel for the ball, it follows that $\int_{D \backslash B} h(y) d y<\infty$ for every ball $B$ contained in $D$. Since $h$ is bounded on $B$, it follows that $h \in L^{1}(D)$. By use of the boundedness and the joint continuity of $(x, y) \mapsto p^{D}(t, x, y)$ (see Ref. 9), it follows by the dominated convergence theorem that $P_{t}^{D} h(x)$ is continuous. For $\alpha=2$, these facts are well known.

In this paper we always assume that $\left(P_{t}^{D}\right)_{t \geq 0}$ is intrinsically ultracontractive, that is, for each $t>0$ there exists a constant $c_{t}$ such that

$$
p^{D}(t, x, y) \leq c_{t} \varphi_{0}(x) \varphi_{0}(y), \quad x, y \in D,
$$

where $\varphi_{0}$ is the positive eigenfunction corresponding to the smallest eigenvalue $\lambda_{0}$ of the Dirichlet Laplacian $\left.(-\Delta)^{\alpha / 2}\right|_{D}$. It is well known that (see, for instance, Ref. 12) when $\left(P_{t}^{D}\right)_{t \geq 0}$ is intrinsically ultracontractive there is $\tilde{c}_{t}>0$ such that

$$
p^{D}(t, x, y) \geq \tilde{c}_{t} \varphi_{0}(x) \varphi_{0}(y), \quad x, y \in D .
$$

Intrinsic ultracontractivity was introduced by Davies and Simon in Ref. 12. It is well known that (see, for instance, Ref. 1), in the case of $\alpha=2,\left(P_{t}^{D}\right)_{t \geq 0}$ is intrinsically ultracontractive when $D$ is a bounded Lipschitz domain, or a Hölder domain of order 0 , or a uniformly Hölder domain of order $\beta \in(0,2)$. The intrinsic ultracontractivity of $\left(P_{t}^{D}\right)_{t \geq 0}$ when $\alpha \in(0,2)$ was first studied in Ref. 9. From Ref. 19 we know that, in the case of $\alpha \in(0,2),\left(P_{t}^{D}\right)_{t \geq 0}$ is intrinsically ultracontractive for any bounded open set $D$ in $\mathbb{R}^{d}$. 
Let $S=\left(S_{t}: t \geq 0\right)$ and $T=\left(T_{t}: t \geq 0\right)$ be two special subordinators. Suppose that $X, S$ and $T$ are independent. We assume that the Laplace exponents of $S$ and $T$, denoted by $\phi$ and $\psi$ respectively, are related by

$$
\lambda=\phi(\lambda) \psi(\lambda) .
$$

We also assume that $\phi$ has the representation (2.2) with $b>0$ or $\mu(0, \infty)=$ $\infty$. We define subordinate processes by

$$
\begin{array}{ll}
Y_{t}^{D}=X^{D}\left(S_{t}\right), & t \geq 0 \\
Z_{t}^{D}=X^{D}\left(T_{t}\right), & t \geq 0 .
\end{array}
$$

Then $Y^{D}=\left(Y_{t}^{D}: t \geq 0\right)$ and $Z^{D}=\left(Z_{t}^{D}: t \geq 0\right)$ are symmetric Hunt processes on $D$. If we use $\rho_{t}(d s)$ and $\theta_{t}(d s)$ to denote the distributions of $S_{t}$ and $T_{t}$ respectively, the semigroups of $Y^{D}$ and $Z^{D}$ are given by

$$
\begin{aligned}
& Q_{t}^{D} f(x)=\int_{0}^{\infty} P_{s}^{D} f(x) \rho_{t}(d s), \\
& R_{t}^{D} f(x)=\int_{0}^{\infty} P_{s}^{D} f(x) \theta_{t}(d s),
\end{aligned}
$$

respectively. The semigroup $Q_{t}^{D}$ has a density given by

$$
q^{D}(t, x, y)=\int_{0}^{\infty} p^{D}(s, x, y) \rho_{t}(d s) .
$$

The semigroup $R_{t}^{D}$ will have a density

$$
r^{D}(t, x, y)=\int_{0}^{\infty} p^{D}(s, x, y) \theta_{t}(d s)
$$

in case $b=0$, while for $b>0, R_{t}^{D}$ is not absolutely continuous with respect to the Lebesgue measure. Let $U$ and $V$ denote the potential measures of $S$ and $T$, respectively. Then there are decreasing functions on $u$ and $v$ defined on $(0, \infty)$ such that $U(d t)=u(t) d t$ and $V(d t)=b \epsilon_{0}(d t)+v(t) d t$. The potential kernels of $Y^{D}$ and $Z^{D}$ are given by

$$
\begin{aligned}
& U^{D} f(x)=\int_{0}^{\infty} P_{t}^{D} f(x) U(d t)=\int_{0}^{\infty} P_{t}^{D} f(x) u(t) d t \\
& V^{D} f(x)=\int_{0}^{\infty} P_{t}^{D} f(x) V(d t)=b f(x)+\int_{0}^{\infty} P_{t}^{D} f(x) v(t) d t
\end{aligned}
$$

respectively. The potential kernel $U^{D}$ has a density given by

$$
U^{D}(x, y)=\int_{0}^{\infty} p^{D}(t, x, y) u(t) d t
$$


while $V^{D}$ need not be absolutely continuous with respect to the Lebesgue measure. For the process $Y^{D}$ we define the potential of a Borel measure $m$ on $D$ by

$$
U^{D} m(x):=\int_{D} U^{D}(x, y) m(d y)=\int_{0}^{\infty} P_{t}^{D} m(x) u(t) d t .
$$

Let $\left(U_{\lambda}^{D}, \lambda>0\right)$ be the resolvent of the semigroup $\left(Q_{t}^{D}, t \geq 0\right)$. Then $U_{\lambda}^{D}$ is given by a kernel which is absolutely continuous with respect to the Lebesgue measure. Moreover, one can easily show that for a bounded Borel function $f$ vanishing outside a compact subset of $D$, the functions $x \mapsto U_{\lambda}^{D} f(x), \lambda>0$, and $x \mapsto U^{D} f(x)$ are continuous. This implies (e.g., Ref. 6, p.266) that excessive functions of $Y^{D}$ are lower semicontinuous.

The factorization in the next proposition is similar in spirit to Theorem 4.1 (5) in Ref. 22. have

Proposition 3.1. (a) For any nonnegative Borel function $f$ on $D$ we

$$
U^{D} V^{D} f(x)=V^{D} U^{D} f(x)=G^{D} f(x), \quad x \in D .
$$

(b) For any Borel measure $m$ on $D$ we have

$$
V^{D} U^{D} m(x)=G^{D} m(x)
$$

Proof. (a) We are only going to show that $U^{D} V^{D} f(x)=G^{D} f(x)$ for all $x \in D$. For the proof of $V^{D} U^{D} f(x)=G^{D} f(x)$ see part (b). For any nonnegative Borel function $f$ on $D$, by using the Markov property and Theorem 2.5 we get that

$$
\begin{aligned}
U^{D} V^{D} f(x) & =\int_{0}^{\infty} P_{t}^{D} V^{D} f(x) u(t) d t \\
& =\int_{0}^{\infty} P_{t}^{D}\left(b f(x)+\int_{0}^{\infty} P_{s}^{D} f(x) v(s) d s\right) u(t) d t \\
& =b U^{D} f(x)+\int_{0}^{\infty} P_{t}^{D}\left(\int_{0}^{\infty} P_{s}^{D} f(x) v(s) d s\right) u(t) d t \\
& =b U^{D} f(x)+\int_{0}^{\infty} \int_{0}^{\infty} P_{t+s}^{D} f(x) v(s) d s u(t) d t
\end{aligned}
$$




$$
\begin{aligned}
& =b U^{D} f(x)+\int_{0}^{\infty} \int_{t}^{\infty} P_{r}^{D} f(x) v(r-t) d r u(t) d t \\
& =b U^{D} f(x)+\int_{0}^{\infty}\left(\int_{0}^{r} u(t) v(r-t) d t\right) P_{r}^{D} f(x) d r \\
& =\int_{0}^{\infty}\left(b u(r)+\int_{0}^{r} u(t) v(r-t) d t\right) P_{r}^{D} f(x) d r \\
& =\int_{0}^{\infty} P_{r}^{D} f(x) d r=G^{D} f(x) .
\end{aligned}
$$

(b) Similarly as above,

$$
\begin{aligned}
V^{D} U^{D} m(x) & =b U^{D} m(x)+\int_{0}^{\infty} P_{t}^{D} U^{D} m(x) v(t) d t \\
& =b U^{D} m(x)+\int_{0}^{\infty} P_{t}^{D}\left(\int_{0}^{\infty} P_{s}^{D} m(x) u(s) d s\right) v(t) d t \\
& =b U^{D} m(x)+\int_{0}^{\infty} \int_{0}^{\infty} P_{t+s}^{D} m(x) u(s) d s v(t) d t \\
& =b U^{D} m(x)+\int_{0}^{\infty} \int_{r}^{\infty} P_{r}^{D} m(x) u(r-t) d r v(t) d t \\
& =b U^{D} m(x)+\int_{0}^{\infty}\left(\int_{0}^{r} u(r-t) v(t) d t\right) P_{r}^{D} m(x) d r \\
& =\int_{0}^{\infty}\left(b+\int_{0}^{r} u(r-t) v(t) d t\right) P_{r}^{D} m(x) d r \\
& =\int_{0}^{\infty} P_{r}^{D} m(x) d r=G^{D} m(x)
\end{aligned}
$$

Proposition 3.2. Let $g$ be an excessive function for $Y^{D}$. Then $V^{D} g$ is excessive for $X^{D}$.

Proof. We first observe that if $g$ is excessive with respect to $Y^{D}$, then $g$ is the increasing limit of $U^{D} f_{n}$ for some $f_{n}$. Hence it follows from Proposition 3.1 that

$$
V^{D} g=\lim _{n \rightarrow \infty} V^{D} U^{D} f_{n}=\lim _{n \rightarrow \infty} G^{D} f_{n}
$$

which implies that $V^{D} g$ is either identically infinite or excessive with respect to $X^{D}$. We prove now that $V^{D} g$ is not identically infinite. In fact, since $g$ is excessive with respect to $Y^{D}$, there exists $x_{0} \in D$ such that for every $t>0$, 


$$
\infty>g\left(x_{0}\right) \geq Q_{t}^{D} g\left(x_{0}\right)=\int_{0}^{\infty} P_{s}^{D} g\left(x_{0}\right) \rho_{t}(d s) .
$$

Thus there is $s>0$ such that $P_{s}^{D} g\left(x_{0}\right)$ is finite. Hence

$$
\infty>P_{s}^{D} g\left(x_{0}\right)=\int_{D} p^{D}\left(s, x_{0}, y\right) g(y) d y \geq \tilde{c}_{s} \varphi_{0}\left(x_{0}\right) \int_{D} \varphi_{0}(y) g(y) d y,
$$

so we have $\int_{D} \varphi_{0}(y) g(y) d y<\infty$. Since $\left(P_{t}^{D}\right)_{t \geq 0}$ is intrinsically ultracontractive, by Theorem 4.2 .5 of Ref. 11 there exists $T>0$ such that

$$
p^{D}(t, x, y) \leq \frac{3}{2} e^{-\lambda_{0} t} \varphi_{0}(x) \varphi_{0}(y), \quad t \geq T, x, y \in D
$$

Consequently

$$
\begin{aligned}
\int_{D} V^{D} g(x) \varphi_{0}(x) d x & =\int_{D} g(x) V^{D} \varphi_{0}(x) d x \\
& =\int_{D} g(x)\left(b \varphi_{0}(x)+\int_{0}^{\infty} P_{t}^{D} \varphi_{0}(x) v(t) d t\right) d x \\
& =\int_{D} g(x)\left(b \varphi_{0}(x)+\int_{0}^{\infty} e^{-\lambda_{0} t} \varphi_{0}(x) v(t) d t\right) d x \\
& =\int_{D} \varphi_{0}(x) g(x) d y\left(b+\int_{0}^{\infty} e^{-\lambda_{0} t} v(t) d t\right)<\infty .
\end{aligned}
$$

Therefore $s=V^{D} g$ is not identically infinite in $D$.

Remark 3.3. Note that the proposition above is valid with $Y^{D}$ and $Z^{D}$ interchanged: if $g$ is excessive for $Z^{D}$, then $U^{D} g$ is excessive for $X^{D}$. Using this we can easily get the following simple fact: if $f$ and $g$ are two nonnegative Borel functions on $D$ such that $V^{D} f$ and $V^{D} g$ are not identically infinite, and that $V^{D} f=V^{D} g$ a.e., then $f=g$ a.e. In fact, since $V^{D} f$ and $V^{D} g$ are excessive for $Z^{D}$, we know that $G^{D} f=U^{D} V^{D} f$ and $G^{D} g=$ $U^{D} V^{D} g$ are excessive for $X^{D}$. Moreover, by the absolute continuity of $U^{D}$, we have that $G^{D} f=G^{D} g$. The a.e. equality of $f$ and $g$ follows from the uniqueness principle for $G^{D}$.

The second part of Proposition 3.1 shows that if $s=G^{D} m$ is the potential of a measure, then $s=V^{D} g$ where $g=U^{D} m$ is excessive for $Y^{D}$. 
The function $g$ can be written in the following way:

$$
\begin{aligned}
g(x) & =\int_{0}^{\infty} P_{s}^{D} m(x) u(s) d s \\
& =\int_{0}^{\infty} P_{s}^{D} m(x)\left(u(\infty)+\int_{s}^{\infty}-d u(t)\right) d s \\
& =\int_{0}^{\infty} P_{s}^{D} m(x) u(\infty) d s+\int_{0}^{\infty} P_{s}^{D} m(x)\left(\int_{s}^{\infty}-d u(t)\right) d s \\
& =u(\infty) s(x)+\int_{0}^{\infty}\left(\int_{0}^{t} P_{s}^{D} m(x) d s\right)(-d u(t)) \\
& =u(\infty) s(x)+\int_{0}^{\infty}\left(P_{t}^{D} s(x)-s(x)\right) d u(t)
\end{aligned}
$$

In the next proposition we will show that every excessive function $s$ for $X^{D}$ can be represented as a potential $V^{D} g$, where $g$, given by (3.2), is excessive for $Y^{D}$. This result was first stated in Ref. 16 as Theorem 2 for the case of stable subordinators and used in Ref. 15. However, the proof given in Ref. 16 does not seem to be complete because of the following two reasons. First, it is only shown that $g$ is almost everywhere equal to an excessive function, while for later applications it is essential that $g$ itself is excessive. Secondly, the use of Lemma 1 in that proof does not seem to be justified. We therefore give a complete proof here which is based on the approach in Ref. 16.

We need the following important lemma.

Lemma 3.4. Let $h$ be a nonnegative harmonic function for $X^{D}$, and let

$$
g(x)=u(\infty) h(x)+\int_{0}^{\infty}\left(P_{t}^{D} h(x)-h(x)\right) d u(t) .
$$

Then $g$ is continuous.

Proof. We only give the proof in the case when $\alpha \in(0,2)$, the proof in the case $\alpha=2$ is similar and essentially given in Ref. 16 . Since the first term in the formula (3.3) is continuous, we have to prove that the second term is also continuous. Let us extend $h$ to $\mathbb{R}^{d} \backslash D$ by setting $h(x)=0$ for all $x \in \mathbb{R}^{d} \backslash D$. This extended $h$ is harmonic for $X$ in $D$.

For any $\epsilon>0$ we have

$$
\left|\int_{\epsilon}^{\infty} d u(t)\right| \leq u(\epsilon)
$$


We first note that from continuity of $h$ and $P_{t}^{D} h$ it follows by the dominated convergence theorem that the function

$$
x \mapsto \int_{\epsilon}^{\infty}\left(P_{t}^{D} h(x)-h(x)\right) d u(t), \quad x \in D,
$$

is continuous. Hence we only need to prove that the function

$$
x \mapsto \int_{0}^{\epsilon}\left(P_{t}^{D} h(x)-h(x)\right) d u(t), \quad x \in D,
$$

is continuous. For any $x_{0} \in D$ choose $r>0$ such that $B\left(x_{0}, 5 r\right) \subset D$. Put $B_{i}=B\left(x_{0}, i r\right)$ for $i=1, \ldots, 4$, and let $\tau_{B_{i}}$ be the exit time of $X$ from $B_{i}$, $i=1, \ldots, 4$. It is enough to show that

$$
\lim _{\epsilon \downarrow 0} \int_{0}^{\epsilon}\left(P_{t}^{D} h(x)-h(x)\right) d u(t)=0
$$

uniformly on $\overline{B_{1}}$. For any $x \in B_{2}, h\left(X_{t \wedge \tau_{B_{2}}}\right)$ is a $\mathbb{P}_{x}$-martingale. Therefore,

$$
\begin{aligned}
0 \leq & h(x)-P_{t}^{D} h(x)=\mathbb{E}_{x}\left[h\left(X_{t \wedge \tau_{B_{2}}}\right)\right]-\mathbb{E}_{x}\left[h\left(X_{t}\right), t<\tau_{D}\right] \\
= & \mathbb{E}_{x}\left[h\left(X_{t}\right), t<\tau_{B_{2}}\right]+\mathbb{E}_{x}\left[h\left(X_{\tau_{B_{2}}}\right), \tau_{B_{2}} \leq t\right] \\
& -\mathbb{E}_{x}\left[h\left(X_{t}\right), t<\tau_{B_{2}}\right]-\mathbb{E}_{x}\left[h\left(X_{t}\right), \tau_{B_{2}} \leq t<\tau_{D}\right] \\
= & \mathbb{E}_{x}\left[h\left(X_{\tau_{B_{2}}}\right), \tau_{B_{2}} \leq t\right]-\mathbb{E}_{x}\left[h\left(X_{t}\right), \tau_{B_{2}} \leq t<\tau_{D}\right] \\
\leq & \mathbb{E}_{x}\left[h\left(X_{\tau_{B_{2}}}\right), \tau_{B_{2}} \leq t\right] \\
= & \mathbb{E}_{x}\left[h\left(X_{\tau_{B_{2}}}\right) 1_{\left\{X\left(\tau_{B_{2}}\right) \in B_{4}\right\}}, \tau_{B_{2}} \leq t\right]+\mathbb{E}_{x}\left[h\left(X_{\tau_{B_{2}}}\right) 1_{\left\{X\left(\tau_{B_{2}}\right) \in B_{4}^{c}\right\}}, \tau_{B_{2}} \leq t\right]
\end{aligned}
$$

Since $h$ is continuous, there exists a constant $M>0$ such that $h(y) \leq M$ for all $y \in B_{4}$. Therefore, we get

$$
\mathbb{E}_{x}\left[h\left(X_{\tau_{B_{2}}}\right) 1_{\left\{X\left(\tau_{B_{2}}\right) \in B_{4}\right\}}, \tau_{B_{2}} \leq t\right] \leq M \mathbb{P}_{x}\left(\tau_{B_{2}} \leq t\right) .
$$

For every $x \in \overline{B_{1}}$, we have

$$
\left\{\tau_{B_{2}} \leq t\right\} \subset\left\{\sup _{0 \leq s \leq t}\left|X_{s}-x\right| \geq r\right\}
$$

almost surely with respect to $\mathbb{P}_{x}$. Therefore one can easily show (see, for instance, Lemma 3.1 of Ref. 25) that there exists $c_{1}>0$ such that

$$
\mathbb{P}_{x}\left(\tau_{B_{2}} \leq t\right) \leq c_{1} t, \quad x \in \overline{B_{1}} .
$$

Thus we have shown that

$$
\mathbb{E}_{x}\left[h\left(X_{\tau_{B_{2}}}\right) 1_{\left\{X\left(\tau_{B_{2}}\right) \in B_{4}\right\}}, \tau_{B_{2}} \leq t\right] \leq c_{1} M t, \quad x \in \overline{B_{1}} .
$$


Now let us deal with the term $\mathbb{E}_{x}\left[h\left(X_{\tau_{B_{2}}}\right) 1_{\left\{X\left(\tau_{B_{2}}\right) \in B_{4}^{c}\right\}}, \tau_{B_{2}} \leq t\right]$. Using the definition of harmonicity and the explicit formula for the Poisson kernel for a ball we can see that $c_{2}:=\int_{D \backslash B_{4}} h(y) d y$ is finite. Therefore for every $x \in \overline{B_{1}}$,

$$
\begin{aligned}
\mathbb{E}_{x} & {\left[h\left(X_{\tau_{B_{2}}}\right) 1_{\left\{X\left(\tau_{B_{2}}\right) \in B_{4}^{c}\right\}}, \tau_{B_{2}} \leq t\right] } \\
& \leq C(d, \alpha) \mathbb{E}_{x} \int_{0}^{\tau_{B_{2}} \wedge t} \int_{D \backslash B_{4}} \frac{h(y)}{\left|X_{s}-y\right|^{d+\alpha}} d y d s \\
& \leq c_{2} c_{3} C(d, \alpha) t .
\end{aligned}
$$

Hence we have

$$
0 \leq h(x)-P_{t}^{D} h(x) \leq c_{4} t, \quad x \in \overline{B_{1}} .
$$

Therefore we have for every $x \in \overline{B_{1}}$,

$$
\left|\int_{0}^{\epsilon}\left(P_{t}^{D} h-h\right)(x) d u(t)\right| \leq c_{4}\left|\int_{0}^{\epsilon} t d u(t)\right| .
$$

By use of (2.14) we get that

$$
\lim _{\epsilon \downarrow 0} \int_{0}^{\epsilon}\left(P_{t}^{D} h(x)-h(x)\right) d u(t)=0
$$

uniformly on $\overline{B_{1}}$. The proof is now complete.

Proposition 3.5. If $s$ is an excessive function with respect to $X^{D}$, then

$$
s(x)=V^{D} g(x), \quad x \in D,
$$

where $g$ is the excessive function for $Y^{D}$ given by the formula

$$
\begin{aligned}
g(x) & =u(\infty) s(x)+\int_{0}^{\infty}\left(P_{t}^{D} s(x)-s(x)\right) d u(t) \\
& =\psi(0) s(x)+\int_{0}^{\infty}\left(s(x)-P_{t}^{D} s(x)\right) d v(t) .
\end{aligned}
$$

Proof. We know that the result is true when $s$ is the potential of a measure. Let $s$ be an arbitrary excessive function of $X^{D}$. By the Riesz decomposition theorem (see, for instance, Chapter 6 of Ref. 6), $s=G^{D} m+$ $h$, where $m$ is a measure on $D$, and $h$ is a nonnegative harmonic function for $X^{D}$. By linearity, it suffices to prove the result for nonnegative harmonic functions. 
In the rest of the proof we assume therefore that $s$ is a nonnegative harmonic function for $X^{D}$. Define the function $g$ by formula (3.4). We have to prove that $g$ is excessive for $Y^{D}$ and $s=V^{D} g$. By Lemma 3.4, we know that $g$ is continuous.

Further, since $s$ is excessive, there exists a sequence of nonnegative functions $f_{n}$ such that $s_{n}:=G^{D} f_{n}$ increases to $s$. Then also $P_{t}^{D} s_{n} \uparrow P_{t}^{D} s$, implying $s_{n}-P_{t}^{D} s_{n} \rightarrow s-P_{t}^{D} s$. If

$$
g_{n}=u(\infty) s_{n}+\int_{0}^{\infty}\left(s_{n}-P_{t}^{D} s_{n}\right)(-d u(t)),
$$

then we know that $s_{n}=V^{D} g_{n}$ and $g_{n}$ is excessive for $Y^{D}$. By use of Fatou's lemma we get that

$$
\begin{aligned}
g & =u(\infty) s+\int_{0}^{\infty}\left(s-P_{t}^{D} s\right)(-d u(t)) \\
& =\lim _{n} u(\infty) s_{n}+\int_{0}^{\infty} \lim _{n}\left(s_{n}-P_{t}^{D} s_{n}\right)(-d u(t)) \\
& \leq \liminf _{n}\left(u(\infty) s_{n}+\int_{0}^{\infty}\left(s_{n}-P_{t}^{D} s_{n}\right)(-d u(t))\right) \\
& =\liminf _{n} g_{n} .
\end{aligned}
$$

This implies (again by Fatou's lemma) that

$$
\begin{aligned}
V^{D} g & \leq V^{D}\left(\liminf g_{n}\right) \\
& \leq \liminf V^{D} g_{n}=\liminf _{n} s_{n}=s
\end{aligned}
$$

For any nonnegative function $f$, put $G_{1}^{D} f(x):=\int_{0}^{\infty} e^{-t} P_{t}^{D} f(x) d t$. Using the excessivity of $s$, we can easily check that $s^{1}:=s-G_{1}^{D} s$ is an excessive function of $X^{D}$. Using an argument similar to that of the proof of Proposition 3.2 we can show that $G^{D} s$ is not identically infinite. Thus by the resolvent equation we get $G^{D} s^{1}=G^{D} s-G^{D} G_{1}^{D} s=G_{1}^{D} s$, or equivalently,

$$
s(x)=s^{1}(x)+G_{1}^{D} s(x)=s^{1}(x)+G^{D} s^{1}(x), \quad x \in D,
$$

By use of formula (3.2) for the potential $G^{D} s_{1}$ and the easy fact that $V^{D}$ and $G_{1}^{D}$ commute, we have 


$$
\begin{aligned}
G_{1}^{D} s & =G^{D} s^{1}=V^{D}\left(u(\infty) G^{D} s^{1}+\int_{0}^{\infty}\left(P_{t}^{D} G^{D} s^{1}-G^{D} s^{1}\right) d u(t)\right) \\
& =V^{D}\left(u(\infty) G_{1}^{D} s+\int_{0}^{\infty}\left(P_{t}^{D} G_{1}^{D} s-G_{1}^{D} s\right) d u(t)\right) \\
& =G_{1}^{D} V^{D}\left(u(\infty) s+\int_{0}^{\infty}\left(P_{t}^{D} s-s\right) d u(t)\right) .
\end{aligned}
$$

By the uniqueness principle it follows that

$$
s=V^{D}\left(u(\infty) s+\int_{0}^{\infty}\left(P_{t}^{D} s-s\right) d u(t)\right)=V^{D} g \text { a.e. in } D .
$$

Together with (3.6), this implies that $V^{D} g=V^{D}\left(\liminf _{n} g_{n}\right)$ a.e. From Remark 3.3 it follows that

$$
g=\liminf _{n} g_{n} \quad \text { a.e. }
$$

By Fatou's lemma and $Y^{D}$-excessiveness of $g_{n}$ we get that,

$$
\lambda U_{\lambda}^{D} g=\lambda U_{\lambda}^{D}\left(\liminf g_{n}\right) \leq \liminf _{n} \lambda U_{\lambda}^{D} g_{n} \leq \liminf g_{n}=g \quad \text { a.e . }
$$

We want to show that, in fact, $\lambda U_{\lambda}^{D} g \leq g$ everywhere, i.e., that $g$ is supermedian. In order to do this we define $\tilde{g}:=\sup _{n \in \mathbb{N}} n U_{n}^{D} g$. Then $\tilde{g} \leq g$ a.e., hence, by the absolute continuity of $U_{n}^{D}, n U_{n}^{D} \tilde{g} \leq n U_{n}^{D} g \leq \tilde{g}$ everywhere. This implies that $\lambda \mapsto \lambda U_{\lambda}^{D} \tilde{g}$ is increasing (see, e.g., Lemma 3.6 in Ref. 5), hence $\tilde{g}$ is supermedian. The same argument gives that $n \mapsto n U_{n}^{D} g$ is increasing a.e. Define

$$
\tilde{\tilde{g}}:=\sup _{\lambda>0} \lambda U_{\lambda}^{D} \tilde{g}=\sup _{n} n U_{n}^{D} \tilde{g} .
$$

Then $\tilde{\tilde{g}}$ is excessive, and therefore lower semicontinuous. Moreover,

$$
\tilde{\tilde{g}}=\sup _{n} n U_{n}^{D} \tilde{g} \leq \tilde{g} \leq g \quad \text { a.e. }
$$

Combining this with the continuity of $g$ and the lower semicontinuity of $\tilde{\tilde{g}}$, we can get that $\tilde{\tilde{g}} \leq g$ everywhere. Further, for $x \in D$ such that $\tilde{g}(x)<\infty$, we have by the monotone convergence theorem and the resolvent equation

$$
\begin{aligned}
\lambda U_{\lambda}^{D} \tilde{g}(x) & =\lim _{n \rightarrow \infty} \lambda U_{\lambda}^{D}\left(n U_{n}^{D}\right) g(x) \\
& =\lim _{n \rightarrow \infty} \frac{n \lambda}{n-\lambda}\left(U_{\lambda}^{D} g(x)-U_{n}^{D} g(x)\right) \\
& =\lambda U_{\lambda}^{D} g(x) .
\end{aligned}
$$


Since $\tilde{g}<\infty$ a.e., we have

$$
\lambda U_{\lambda}^{D} \tilde{g}=\lambda U_{\lambda}^{D} g \quad \text { a.e. }
$$

Together with the definition of $\tilde{g}$ this implies that

$$
\tilde{\tilde{g}}=\tilde{g} \quad \text { a.e. }
$$

By the continuity of $g$ and the fact that the measures $n U_{n}^{D}(x, \cdot)$ converge weakly to the point mass at $x$, we have that for every $x \in D$

$$
g(x) \leq \liminf _{n \rightarrow \infty} n U_{n}^{D} g(x) \leq \tilde{g}(x) .
$$

Hence, by using (3.8), it follows that $g \leq \tilde{\tilde{g}}$ a.e. Since we already proved that $\tilde{\tilde{g}} \leq g$, it holds that $g=\tilde{\tilde{g}}$ a.e. By the absolute continuity of $U_{\lambda}^{D}, g \geq$ $\tilde{\tilde{g}} \geq \lambda U_{\lambda}^{D} \tilde{\tilde{g}}=\lambda U_{\lambda}^{D} g$ everywhere, i.e., $g$ is supermedian.

Since it is well known (see e.g. Ref. 10) that a supermedian function which is lower semicontinuous is in fact excessive, this proves that $g$ is excessive for $Y^{D}$. By Proposition 3.2 we then have that $V^{D} g \leq s$ is excessive for $X^{D}$. Moreover, $V^{D} g=s$ a.e., and both functions being excessive for $X^{D}$, they are equal everywhere.

It remains to notice that the formula (3.5) follows immediately from (3.4) by noting that $u(\infty)=\psi(0)$ and $d u(t)=-d v(t)$.

Propositions 3.1 and 3.5 can be combined in the following theorem containing additional information on harmonic functions.

Theorem 3.6. If $s$ is excessive with respect to $X^{D}$, then there is a function $g$ excessive with respect to $Y^{D}$ such that $s=V^{D} g$. The function $g$ is given by the formula (3.2). Furthermore, if $s$ is harmonic with respect to $X^{D}$, then $g$ is harmonic with respect to $Y^{D}$.

Conversely, if $g$ is excessive with respect to $Y^{D}$, then the function $s$ defined by $s=V^{D} g$ is excessive with respect to $X^{D}$. If, moreover, $g$ is harmonic with respect to $Y^{D}$, then $s$ is harmonic with respect to $X^{D}$.

Every nonnegative harmonic function for $Y^{D}$ is continuous.

Proof. It remains to show the statements about harmonic functions. First note that every excessive functions $g$ for $Y^{D}$ admits the Riesz decomposition $g=U^{D} m+h$ where $m$ is a Borel measure on $D$ and $h$ is harmonic function of $Y^{D}$ (see Chapter 6 of Ref. 6 and note that the assumptions on pp. 265, 266 are satisfied). We have already mentioned that excessive functions of $X^{D}$ admit such a decomposition. Since excessive functions of $X^{D}$ and $Y^{D}$ are in 1-1 correspondence, and since potentials of measures 
of $X^{D}$ and $Y^{D}$ are in 1-1 correspondence, the same must hold for nonnegative harmonic functions of $X^{D}$ and $Y^{D}$.

The continuity of nonnegative harmonic functions for $Y^{D}$ follows from Lemma 3.4 and Proposition 3.5.

It follows from the theorem above that $V^{D}$ is a bijection from $\mathcal{S}\left(Y^{D}\right)$ to $\mathcal{S}\left(X^{D}\right)$, and is also a bijection from $\mathcal{H}^{+}\left(Y^{D}\right)$ to $\mathcal{H}^{+}\left(X^{D}\right)$. We are going to use $\left(V^{D}\right)^{-1}$ to denote the inverse map and so we have for any $s \in$ $\mathcal{S}\left(Y^{D}\right)$

$$
\begin{aligned}
\left(V^{D}\right)^{-1} s(x) & =u(\infty) s(x)+\int_{0}^{\infty}\left(P_{t}^{D} s(x)-s(x)\right) d u(t) \\
& =\psi(0) s(x)+\int_{0}^{\infty}\left(s(x)-P_{t}^{D} s(x)\right) d v(t)
\end{aligned}
$$

Although the map $V^{D}$ is order preserving, we do not know if the inverse map $\left(V^{D}\right)^{-1}$ is order preserving on $\mathcal{S}\left(X^{D}\right)$. However from the formula above we can see that $\left(V^{D}\right)^{-1}$ is order preserving on $\mathcal{H}^{+}\left(X^{D}\right)$.

By combining Proposition 3.1 and Theorem 3.6 we get the following relation which we are going to use later.

Proposition 3.7. For any $x, y \in D$, we have

$$
U^{D}(x, y)=\left(V^{D}\right)^{-1}\left(G^{D}(\cdot, y)\right)(x) .
$$

The continuity of harmonic functions, together with the intrinsic ultracontractivity of the semigroup $\left(P_{t}^{D}\right)$, is sufficient to prove the Harnack inequality for nonnegative harmonic functions for the process $Y^{D}$. Here we will only state two necessary lemmas and the theorem. For arguments of proofs we refer the reader to Section 4 of Ref. 15.

Lemma 3.8. Suppose that $\left(P_{t}^{D}\right)$ is intrinsically ultracontractive. There exists a constant $C>0$ such that

$$
V^{D} s \leq C s, \quad \forall s \in \mathcal{S}\left(Y^{D}\right) .
$$

Lemma 3.9. Suppose $\left(P_{t}^{D}\right)$ is intrinsically ultracontractive. If $s \in$ $\mathcal{S}\left(Y^{D}\right)$, then for any $x \in D$,

$$
s(x) \geq \frac{1}{2 C} e^{-\lambda_{0} T} \frac{1}{\psi\left(\lambda_{0}\right)} \varphi_{0}(x) \int_{D} s(y) \varphi_{0}(y) d y,
$$

where $T$ is the constant in (3.1) and $C$ is the constant in (3.10). 
Theorem 3.10. Suppose that $\left(P_{t}^{D}\right)$ is intrinsically ultracontractive. For any compact subset $K$ of $D$, there exists a constant $C$ depending on $K$ and $D$ such that for any $h \in \mathcal{H}^{+}\left(Y^{D}\right)$,

$$
\sup _{x \in K} h(x) \leq C \inf _{x \in K} h(x) .
$$

\section{MARTIN BOUNDARY OF THE SUBORDINATE PROCESS}

In this section we will always assume the following Assumption A: $D$ is a bounded $\kappa$-fat set for some $\kappa \in(0,1)$ when $\alpha \in(0,2)$, and $D$ is a bounded Lipschitz domain when $\alpha=2$.

Recall (see Ref. 26) that, for $\kappa \in(0,1)$, an open set $D$ in $\mathbb{R}^{d}$ is called a $\kappa$-fat set if there exists $R>0$ such that for every $z \in \partial D$ and $r \in(0, R)$, $D \cap B(z, r)$ contains a ball $B\left(A_{r}(z), \kappa r\right)$.

Fix a point $x_{0} \in D$ and set

$$
M^{D}(x, y)=\frac{G^{D}(x, y)}{G^{D}\left(x_{0}, y\right)}, \quad x, y \in D .
$$

It is well known that the limit

$$
\lim _{D \ni y \rightarrow z} M^{D}(x, y)
$$

exists for every $x \in D$ and $z \in \partial D$. The function $M^{D}(x, z):=\lim _{D \ni y \rightarrow z} M^{D}$ $(x, y)$ on $D \times \partial D$ defined above is called the Martin kernel of $X^{D}$ based at $x_{0}$. The Martin boundary and minimal Martin boundary of $X^{D}$ both coincide with the Euclidean boundary $\partial D$. For these and other results about the Martin boundary of $X^{D}$ in the case $\alpha=2$, one can see Ref. 2; For these and other results about the Martin boundary of $X^{D}$ in the case $\alpha \in$ $(0,2)$, one can see Ref. 26 . One of the goals of this section is to determine the Martin boundary of $Y^{D}$.

By using the Harnack inequality (in the case $\alpha \in(0,2)$, we need to use the version in Ref. 7), one can easily show that (see, for instance, pages 17-18 of Ref. 13), if $\left(h_{j}\right)$ is a sequence of functions in $\mathcal{H}^{+}\left(X^{D}\right)$ converging pointwise to a function $h \in \mathcal{H}^{+}\left(X^{D}\right)$, then $\left(h_{j}\right)$ is locally uniformly bounded in $D$ and equicontinuous at every point in $D$. Using this one can get that, if $\left(h_{j}\right)$ is a sequence of functions in $\mathcal{H}^{+}\left(X^{D}\right)$ converging pointwise to a function $h \in \mathcal{H}^{+}\left(X^{D}\right)$, then $\left(h_{j}\right)$ converges to $h$ uniformly on compact subsets of $D$. We are going to use this fact below.

Lemma 4.1. Suppose that $x_{0} \in D$ is a fixed point. 
(a) Let $\left(x_{j}\right)$ be a sequence of points in $D$ converging to $x \in D$ and let $\left(h_{j}\right)$ be a sequence of functions in $\mathcal{H}^{+}\left(X^{D}\right)$ with $h_{j}\left(x_{0}\right)=1$ for all $j$. If the sequence $\left(h_{j}\right)$ converges to a function $h \in$ $\mathcal{H}^{+}\left(X^{D}\right)$, then for each $t>0$

$$
\lim _{j} P_{t}^{D} h_{j}\left(x_{j}\right)=P_{t}^{D} h(x) .
$$

(b) If $\left(y_{j}, j \geq 1\right)$ is a sequence of points in $D$ such that $\lim _{j} y_{j}=z \in$ $\partial D$, then for each $t>0$ and for each $x \in D$

$$
\lim _{j} P_{t}^{D}\left(\frac{G^{D}\left(\cdot, y_{j}\right)}{G^{D}\left(x_{0}, y_{j}\right)}\right)(x)=P_{t}^{D}\left(M^{D}(\cdot, z)\right)(x) .
$$

Proof. In the case $\alpha=2$, this lemma is just Lemma 5.1 of Ref. 15 . The proof of the case when $\alpha \in(0,2)$ is similar to that of Lemma 5.1 of Ref. 15, only now we use the result on the identification of Martin boundaries for $\kappa$-fat sets in Ref. 26 and the $3 \mathrm{G}$ inequality there. We omit the details.

Theorem 4.2. Suppose that $x_{0} \in D$ is a fixed point.

(a) If $\left(x_{j}\right)$ is a sequence of points in $D$ converging to $x \in D$ and $\left(h_{j}\right)$ is a sequence of functions in $\mathcal{H}^{+}\left(X^{D}\right)$ converging to a function $h \in \mathcal{H}^{+}\left(X^{D}\right)$, then

$$
\lim _{j}\left(V^{D}\right)^{-1} h_{j}\left(x_{j}\right)=\left(V^{D}\right)^{-1} h(x) .
$$

(b) If $\left(y_{j}\right)$ is a sequence of points in $D$ converging to $z \in \partial D$, then for every $x \in D$,

$$
\begin{aligned}
\lim _{j}\left(V^{D}\right)^{-1}\left(\frac{G^{D}\left(\cdot, y_{j}\right)}{G^{D}\left(x_{0}, y_{j}\right)}\right)(x) & =\lim _{j} \frac{\left(V^{D}\right)^{-1}\left(G^{D}\left(\cdot, y_{j}\right)\right)(x)}{G^{D}\left(x_{0}, y_{j}\right)} \\
& =\left(V^{D}\right)^{-1} M^{D}(\cdot, z)(x) .
\end{aligned}
$$

Proof. We only give the proof in the case when $\alpha \in(0,2)$, the proof in the case $\alpha=2$ is similar.

(a) Normalizing by $h_{j}\left(x_{0}\right)$ if necessary, we may assume without loss of generality that $h_{j}\left(x_{0}\right)=1$ for all $j \geq 1$. Let $\epsilon>0$. We have 


$$
\begin{aligned}
& \left|\left(V^{D}\right)^{-1} h_{j}\left(x_{j}\right)-\left(V^{D}\right)^{-1} h(x)\right| \\
& =\mid \int_{0}^{\infty}\left(P_{t}^{D} h_{j}\left(x_{j}\right)-h_{j}\left(x_{j}\right) d u(t)-\int_{0}^{\infty}\left(P_{t}^{D} h(x)-h(x)\right) d u(t)+u(\infty)\left(h_{j}\left(x_{j}\right)\right.\right. \\
& \quad-h(x)) \mid \leq \int_{0}^{\epsilon}\left(P_{t}^{D} h_{j}\left(x_{j}\right)-h_{j}\left(x_{j}\right)\right) d u(t)+\int_{0}^{\epsilon}\left(P_{t}^{D} h(x)-h(x)\right) d u(t) \\
& \quad+\left|\int_{\epsilon}^{\infty}\left(P_{t}^{D} h_{j}\left(x_{j}\right)-h_{j}\left(x_{j}\right)\right) d u(t)-\int_{\epsilon}^{\infty}\left(P_{t}^{D} h(x)-h(x)\right) d u(t)\right| \\
& \quad+u(\infty)\left|h_{j}\left(x_{j}\right)-h(x)\right| .
\end{aligned}
$$

The last term clearly converges to zero as $j \rightarrow \infty$.

For any $x \in D$ choose $r>0$ such that $B(x, 5 r) \subset D$. Put $B_{i}=B(x, i r)$ for $i=1, \ldots, 4$. Without loss of generality we may and do assume that $x_{j} \in B_{1}$ for all $j \geq 1$. Similarly as in the proof of Lemma 3.4, we extend functions $h$ and $h_{j}$ to be identically zero outside $D$. Since $h$ and $h_{j}$ are continuous in $D$ and $\left(h_{j}\right)$ is locally uniformly bounded in $D$, there is a constant $M>0$ such that $h$ and $h_{j}, j=1,2, \ldots$, are all bounded from above by $M$ on $B_{4}$. Now from the proof of Lemma 3.4 we know that there is a constant $c_{1}>0$ such that

$$
\mathbb{E}_{y}\left[h\left(X_{\tau_{B_{2}}}\right) 1_{\left\{X\left(\tau_{B_{2}}\right) \in B_{4}\right\}}, \tau_{B_{2}} \leq t\right] \leq c_{1} M t, \quad y \in \overline{B_{1}},
$$

and

$$
\mathbb{E}_{y}\left[h_{j}\left(X_{\tau_{B_{2}}}\right) 1_{\left\{X\left(\tau_{B_{2}}\right) \in B_{4}\right\}}, \tau_{B_{2}} \leq t\right] \leq c_{1} M t, \quad y \in \overline{B_{1}}, j \geq 1 .
$$

Using the boundedness of $\left(h_{j}(x)\right)$, the definition of harmonicity and the explicit formula for the Poisson kernel of a ball one can show that

$$
c_{2}:=\left(\int_{D \backslash B_{4}} h(z) d z\right) \vee\left(\sup _{j} \int_{D \backslash B_{4}} h_{j}(z) d z\right)<\infty .
$$

Therefore we have for every $y \in \overline{B_{1}}$,

$$
\begin{aligned}
& \mathbb{E}_{y}\left[h\left(X_{\tau_{B_{2}}}\right) 1_{\left\{X\left(\tau_{B_{2}}\right) \in B_{4}^{c}\right\}}, \tau_{B_{2}} \leq t\right] \\
& \leq C(d, \alpha) \mathbb{E}_{y} \times \int_{0}^{\tau_{B_{2}} \wedge t} \int_{D \backslash B_{4}} \frac{h(y)}{\left|X_{s}-y\right|^{d+\alpha}} d y d s \leq c_{2} c_{3} C(d, \alpha) t,
\end{aligned}
$$

and for every $j \geq 1$,

$$
\begin{aligned}
& \mathbb{E}_{y}\left[h_{j}\left(X_{\tau_{B_{2}}}\right) 1_{\left\{X\left(\tau_{B_{2}}\right) \in B_{4}^{c}\right\}}, \tau_{B_{2}} \leq t\right] \\
& \leq C(d, \alpha) \mathbb{E}_{y} \times \int_{0}^{\tau_{B_{2}} \wedge t} \int_{D \backslash B_{4}} \frac{h_{j}(y)}{\left|X_{s}-y\right|^{d+\alpha}} d y d s \leq c_{2} c_{3} C(d, \alpha) t .
\end{aligned}
$$


Hence we have

$$
0 \leq h(y)-P_{t}^{D} h(y) \leq c_{4} t, \quad y \in \overline{B_{1}},
$$

and

$$
0 \leq h_{j}(y)-P_{t}^{D} h_{j}(y) \leq c_{4} t, \quad y \in \overline{B_{1}}, j \geq 1 .
$$

Therefore we have,

$$
\left|\int_{0}^{\epsilon}\left(P_{t}^{D} h-h\right)(y) d u(t)\right| \leq c_{4}\left|\int_{0}^{\epsilon} t d u(t)\right|, \quad y \in \overline{B_{1}}
$$

and

$$
\left|\int_{0}^{\epsilon}\left(P_{t}^{D} h_{j}-h_{j}\right)(y) d u(t)\right| \leq c_{4}\left|\int_{0}^{\epsilon} t d u(t)\right|, \quad y \in \overline{B_{1}}, j \geq 1 .
$$

Using (2.14) we get that

$$
\lim _{\epsilon \downarrow 0} \int_{0}^{\epsilon}\left(P_{t}^{D} h(x)-h(x)\right) d u(t)=0,
$$

and

$$
\lim _{\epsilon \downarrow 0} \int_{0}^{\epsilon}\left(P_{t}^{D} h_{j}\left(x_{j}\right)-h_{j}\left(x_{j}\right)\right) d u(t)=0,
$$

Further,

$$
\begin{aligned}
& \left|\int_{\epsilon}^{\infty}\left(P_{t}^{D} h_{j}\left(x_{j}\right)-h_{j}\left(x_{j}\right)\right) d u(t)-\int_{\epsilon}^{\infty}\left(P_{t}^{D} h(x)-h(x)\right) d u(t)\right| \\
& \leq \int_{\epsilon}^{\infty}\left(\left|h_{j}\left(x_{j}\right)-h\left(x_{j}\right)\right|+\left|h\left(x_{j}\right)-h(x)\right|\right) d u(t) \\
& \quad+\int_{\epsilon}^{\infty}\left|P_{t}^{D} h_{j}\left(x_{j}\right)-P_{t}^{D} h(x)\right| d u(t) .
\end{aligned}
$$

Since $\left|h_{j}\left(x_{j}\right)-h\left(x_{j}\right)\right|+\left|h\left(x_{j}\right)-h(x)\right| \leq 2 M$ and $\left|P_{t}^{D} h_{j}\left(x_{j}\right)-P_{t}^{D} h(x)\right| \leq M$ for all $j \geq 1$ and all $x \in \overline{B_{1}}$, we can apply Lemma 4.1(a) and the dominated convergence theorem to get

$$
\lim _{j} \int_{\epsilon}^{\infty}\left(\left|h_{j}\left(x_{j}\right)-h\left(x_{j}\right)\right|+\left|h\left(x_{j}\right)-h(x)\right|\right) d u(t)=0
$$

and

$$
\lim _{j} \int_{\epsilon}^{\infty}\left|P_{t}^{D} h_{j}\left(x_{j}\right)-P_{t}^{D} h(x)\right| v(t)=0
$$


The proof of (a) is now complete. (b) The proof of (b) is similar to (a). The only difference is that we use 4.1(b) in this case. We omit the details.

Let us define the function $K_{Y}^{D}(x, z):=\left(V^{D}\right)^{-1} M^{D}(\cdot, z)(x)$ on $D \times \partial D$. For each fixed $z \in \partial D, K_{Y}^{D}(\cdot, z) \in \mathcal{H}^{+}\left(Y^{D}\right)$. By the first part of Theorem 4.2, we know that $K_{Y}^{D}(x, z)$ is continuous on $D \times \partial D$. Let $\left(y_{j}\right)$ be a sequence of points in $D$ converging to $z \in \partial D$, then from Theorem 4.2(b) we get that

$$
\begin{aligned}
K_{Y}^{D}(x, z) & =\lim _{j \rightarrow \infty}\left(V^{D}\right)^{-1}\left(\frac{G^{D}\left(\cdot, y_{j}\right)}{G^{D}\left(x_{0}, y_{j}\right)}\right)(x) \\
& =\lim _{j \rightarrow \infty} \frac{\left(V^{D}\right)^{-1}\left(G^{D}\left(\cdot, y_{j}\right)\right)(x)}{G^{D}\left(x_{0}, y_{j}\right)} \\
& =\lim _{j \rightarrow \infty} \frac{U^{D}\left(x, y_{j}\right)}{G^{D}\left(x_{0}, y_{j}\right)}
\end{aligned}
$$

where the last line follows from Proposition 3.7. In particular, there exists the limit

$$
\lim _{j \rightarrow \infty} \frac{U^{D}\left(x_{0}, y_{j}\right)}{G^{D}\left(x_{0}, y_{j}\right)}=K_{Y}^{D}\left(x_{0}, z\right) .
$$

Now we define a function $M_{Y}^{D}$ on $D \times \partial D$ by

$$
M_{Y}^{D}(x, z):=\frac{K_{Y}^{D}(x, z)}{K_{Y}^{D}\left(x_{0}, z\right)}, \quad x \in D, z \in \partial D .
$$

For each $z \in \partial D, M_{Y}^{D}(\cdot, z) \in \mathcal{H}_{+}\left(Y^{D}\right)$. Moreover, $M_{Y}^{D}$ is jointly continuous on $D \times \partial D$. From the definition above and (4.1) we can easily see that

$$
\lim _{D \ni y \rightarrow z} \frac{U^{D}(x, y)}{U^{D}\left(x_{0}, y\right)}=M_{Y}^{D}(x, z), \quad x \in D, z \in \partial D .
$$

Theorem 4.3. The Martin boundary and the minimal Martin boundary of $Y^{D}$ both coincide with the Euclidean boundary $\partial D$, and the Martin kernel based at $x_{0}$ is given by the function $M_{Y}^{D}$.

Proof. The fact that $M_{Y}^{D}$ is the Martin kernel of $Y^{D}$ based at $x_{0}$ has been proven in the paragraph above. It follows from Theorem 3.6 that when $z_{1}$ and $z_{2}$ are two distinct points on $\partial D$, the functions $M_{Y}^{D}\left(\cdot, z_{1}\right)$ and $M_{Y}^{D}\left(\cdot, z_{2}\right)$ are not identical. Therefore the Martin boundary of $Y^{D}$ coincides with the Euclidean boundary $\partial D$. Since $M^{D}(\cdot, z) \in \mathcal{H}^{+}\left(X^{D}\right)$ 
is minimal, by the order preserving property of $\left(Y^{D}\right)^{-1}$ we know that $M_{Y}^{D}(\cdot, z) \in \mathcal{H}^{+}\left(Y^{D}\right)$ is also minimal. Therefore the minimal Martin boundary of $Y_{D}$ also coincides with the Euclidean boundary $\partial D$.

It follows from Theorem 4.3 and the general theory of Martin boundary that for any $g \in \mathcal{H}^{+}\left(Y^{D}\right)$ there exists a finite measure $n$ on $\partial D$ such that

$$
g(x)=\int_{\partial D} M_{Y}^{D}(x, z) n(d z), \quad x \in D .
$$

The measure $n$ is sometimes called the Martin measure of $g$. The following result gives the relation between the Martin measure of $h \in \mathcal{H}^{+}\left(X^{D}\right)$ and the Martin measure of $\left(V^{D}\right)^{-1} h \in \mathcal{H}^{+}\left(Y^{D}\right)$.

Proposition 4.4. If $h \in \mathcal{H}^{+}\left(X^{D}\right)$ has the representation

$$
h(x)=\int_{\partial D} M^{D}(x, z) m(d z), \quad x \in D,
$$

then

$$
\left(V^{D}\right)^{-1} h(x)=\int_{\partial D} M_{Y}^{D}(x, z) n(d z), \quad x \in D
$$

with $n(d z)=K_{Y}^{D}\left(x_{0}, z\right) m(d z)$.

Proof. By assumption we know that

$$
h(x)=\int_{\partial D} M^{D}(x, z) m(d z), \quad x \in D .
$$

Using (3.4) and Fubini's theorem we get

$$
\begin{aligned}
\left(V^{D}\right)^{-1} h(x) & =\int_{\partial D}\left(V^{D}\right)^{-1}\left(M^{D}(\cdot, z)\right)(x) m(d z) \\
& =\int_{\partial D} M_{Y}^{D}(x, z) K_{Y}^{D}\left(x_{0}, z\right) m(d z) \\
& =\int_{\partial D} M_{Y}^{D}(x, z) n(d z),
\end{aligned}
$$

with $n(d z)=K_{Y}^{D}\left(x_{0}, z\right) m(d z)$. The proof is now complete.

From Theorem 4.2 we know that $\left(V^{D}\right)^{-1}: \mathcal{H}^{+}\left(X^{D}\right) \rightarrow \mathcal{H}^{+}\left(Y^{D}\right)$ is continuous with respect to topologies of locally uniform convergence. In the next result we show that $V^{D}: \mathcal{H}^{+}\left(Y^{D}\right) \rightarrow \mathcal{H}^{+}\left(X^{D}\right)$ is also continuous. 
Proposition 4.5. Let $\left(g_{j}, j \geq 0\right)$ be a sequence of functions in $\mathcal{H}^{+}\left(Y^{D}\right)$ converging pointwise to the function $g \in \mathcal{H}^{+}\left(Y^{D}\right)$. Then $\lim _{j \rightarrow \infty} V^{D} g_{j}(x)=$ $V^{D} g(x)$ for every $x \in D$.

Proof. Without loss of generality we may assume that $g_{j}\left(x_{0}\right)=1$ for all $j \in \mathbb{N}$. Then there exist probability measures $n_{j}, j \in \mathbb{N}$, and $n$ on $\partial D$ such that $g_{j}(x)=\int_{\partial D} M_{Y}^{D}(x, z) n_{j}(d z), j \in \mathbb{N}$, and $g(x)=\int_{\partial D} M_{Y}^{D}(x, z) n(d z)$. It is easy to show that the convergence of the harmonic functions $h_{j}$ implies that $n_{j} \rightarrow n$ weakly. Let $V^{D} g_{j}(x)=\int_{\partial D} M^{D}(x, z) m_{j}(d z)$ and $V^{D} g(x)=\int_{\partial D} M^{D}(x, z) m(d z)$. Then $n_{j}(d z)=K_{Y}^{D}\left(x_{0}, z\right) m_{j}(d z)$ and $n(d z)=$ $K_{Y}^{D}\left(x_{0}, z\right) m(d z)$. Since the density $K_{Y}^{D}\left(x_{0}, \cdot\right)$ is bounded away from zero and bounded from above, it follows that $m_{j} \rightarrow m$ weakly. From this the claim of proposition follows immediately.

\section{NOTE ADDED IN PROOF}

After this paper was accepted we learned about the paper J. Bertoin (1997), Regenerative embeddings of Markov sets, Probability Theory and Related Fields 108, 559-571. Our Theorem 2.1 is essentially contained in Corollary 1 and Corollary 2 of Bertoin's paper.

\section{REFERENCES}

1. Bañuelos, R. (1991). Intrinsic ultracontractivity and eigenfunction estimates for Schrödinger operators, J. Funct. Anal. 100, 181-206.

2. Bass, R. (1995). Probabilistic Techniques in Analysis, Springer, New York.

3. Bertoin, J. (1996). Lévy Processes, Cambridge University Press, Cambridge.

4. Bertoin, J., and Yor, M. (2001). On subordinators, self-similar Markov processes and some factorizations of the exponential random variable. Elect. Comm. in Probab. 6, 95-106.

5. Bliedtner, J., and Hansen, W. (1986). Potential Theory: An Analytic and Probabilistic Approach to Balayage. Springer, New York.

6. Blumenthal, R. M., and Getoor, R. K. (1968). Markov Processes and Potential Theory. Academic Press, New York.

7. Bogdan, K. (1997). The boundary Harnack principle for the fractional Laplacian. Studia Math. 123, 43-80.

8. Bondesson, L. (1992). Generalized Gamma Convolutions and Related Classes of Distributions and Densities. Springer, New York.

9. Chen, Z.-Q., and Song, R. (1997). Intrinsic ultracontractivity and conditional gauge for symmetric stable processes. J. Funct. Anal. 150, 204-239.

10. Chung, K.-L. (1982). Lectures from Markov Processes to Brownian Motion. Springer, New York.

11. Davies, E. B. (1989). Heat Kernels and Spectral Theory. Cambridge University Press, Cambridge.

12. Davies, E. B., and Simon, B. (1984). Ultracontractivity and the heat kernel for Schrödinger operators and Dirichlet Laplacians. J. Funct. Anal. 59, 335-395. 
13. Doob, J. L. (1984). Classical Potential Theory and its Probabilistic Counterparts Springer, New York.

14. Farkas, W., and Jacob, N. (2001). Sobolev spaces on non-smooth domains and Dirichlet forms related to subordinate reflecting diffusions. Math. Nachr. 224, 75-104.

15. Glover, J., Pop-Stojanovic, Z., Rao, M., Šikić, H., Song, R., and Vondraček, Z. (2004). Harmonic functions of subordinate killed Brownian motions. J. Funct. Anal. 215, 399-426.

16. Glover, J., Rao, M., Šikić, H., and Song, R. (1994). Г-potentials, in Classical and modern potential theory and applications (Chateau de Bonas, 1993), 217-232, Kluwer Acad. Publ., Dordrecht.

17. Jacob, N. (2001). Pseudo Differential Operators and Markov Processes Vol. 1, Imperial College Press, London, 2001.

18. Jacob, N., and Schilling, R. L. (1999). Some Dirichlet spaces obtained by subordinate reflected diffusions. Rev. Mat. Iberoamericana 15, 59-91.

19. Kulczycki, T. (1998). Intrinsic ultracontractivity for symmetric stable processes. Bull. Polish Academy of Sciences 46, 325-334.

20. Nakamura, Y. (1989). Classes of operator monotone functions and Stieltjes functions. In Dym, H. et al., (eds.), The Gohberg Anniversary Collection, Vol. II: Topics in Analysis and Operator Theory, Operator Theory: Advances and Applications, Vol. 41, Birkhäuser, Basel. pp.395-404

21. Rao, M., Song, R., and Vondraček, Z. (2006). Green function estimates and Harnack inequality for subordinate Brownian motions, Potential Anal., 25, 1-27.

22. Schilling, R. L. (1998). Subordination in the sense of Bochner and a related functional calculus. J. Austral. Math. Soc., Ser. A, 64, 368-396.

23. Song, R. (2004). Sharp bounds on the density, Green function and jumping function of subordinate killed BM. Probab. Th. Rel. Fields, 128, 606-628.

24. Song, R., and Vondraček, Z. (2003). Potential Theory of Subordinate Killed Brownian Motion in a Domain. Probab. Th. Rel. Fields 125, 578-592.

25. Song, R., and Vondraček, Z. (2004). Harnack inequalities for some classes of Markov processes. Math. Z. 246, 177-202

26. Song, R., and Wu, J.-M. (1999). Boundary Harnack principle for symmetric stable processes. J. Funct. Anal. 168, 403-427. 